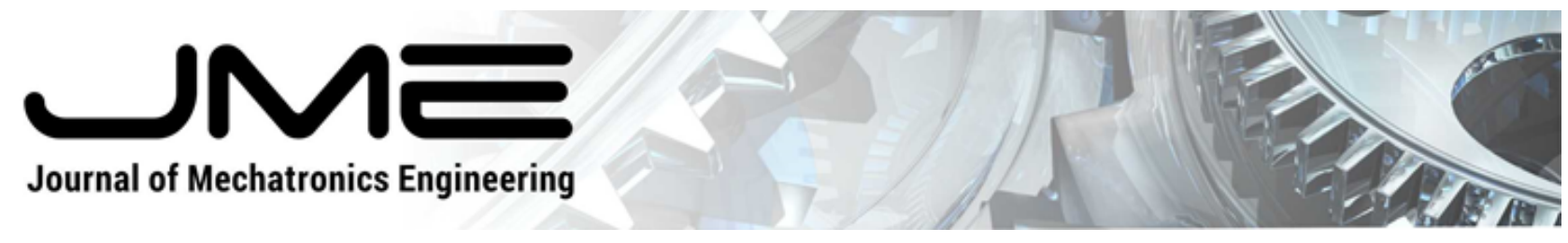

\title{
Development of forward kinematics and construction of the driving and command system of a robotic manipulator
}

\author{
Odilon Linhares Carvalho Mendes ${ }^{1,2}$, Rogério da Silva Oliveira $^{1}$ \\ Instituto Federal do Ceará - IFCE, Campus Fortaleza, \\ ${ }^{1}$ Laboratório de Instrumentação e Robótica - LIR \\ 2 Telecomunications Engineering Program - PPGET \\ Av. Treze de Maio, 2081, \\ Benfica, 60040-215 Fortaleza, Ceará, Brazil \\ <odilonlinharesmendes@gmail.com>, <rogerio@ifce.edu.br>
}

DOI: http://dx.doi.org/10.21439/jme.v3i1.83

Received: 09 Apr. 2020. Accepted: 24 May 2020

\begin{abstract}
The idea of working with a robotic manipulator came from the availability of doing a retrofit of the driving and command system for the RD5 robot, a manipulator that is part of the IFCE's Robotics Laboratory. The challenges of working with this robot are the fact that this manipulator had not been used for some time, and it was necessary adjustments. For example, the robot's joints needed to be tightened, its connections needed to be rewired, and its damaged potentiometers needed to be replaced. The objectives of this work are the development of the Forward Kinematics (FK) and the construction of its driving and command system. The expected positions are obtained from the FK using the DenavitHatenberg (DH) method. The expected positioning of the manipulator in each situation was compared with that obtained through the driving and command system, and, thus, it was possible to perform the acquisition of its positioning error. The acquired position mean error of $0.56 \mathrm{~cm}$ is satisfactory, validating the project.
\end{abstract}

Keywords: Denavit-Hatenberg, Driving System, Forward Kinematic, Retrofit, Robotic Manipulator.

\section{Introduction}

The current society is facing a growing need for efficiency and precision in various tasks. There are complex jobs, which generate risk factors or difficulties that sometimes make it impossible for a person to perform them. Due to these factors and the advancement of technology, humankind developed mechatronic devices to perform such jobs (ROSÁRIO; MAURÍCIO, 2005). Robots are increasingly present in various areas of society. They are providing domestic services, assisting in scientific research, and performing industrial tasks. Technological advancements have driven the development of robotics, which began to emerge in the 1940s with the first patents. Coincidentally, robotics appeared in the same period as computers.
Robots provide advantages, especially in the industry. Some of them are the reduction of the number of employees to perform a task, higher quality products, and the increase in productivity (ROSÁRIO; MAURÍCIO 2005). In medicine, robots can assist in performing surgeries that require a very high level of stability and precision of movements.

Specifically speaking of robotic manipulators, they are composed of rigid links connected by joints, which move in the workspace in order to perform the assignment defined by programming (MITTAL; NAGRATH. 2003 ). Forward Kinematics (FK) of a robotic manipulator is the study of the position and speed of its actuator and joints. Through this, the desired positioning state space is known for any joint angle arrangement. Due to the complexity of modeling the FK of a manipulator, a 
standard convention was created in order to standardize the coordinates of the reference systems for spatial connections. This model is called Denavit-Hatenberg (DH) notation.

A driving and command system of a robotic manipulator can use FK as an auxiliary tool for guidance and reference to know how close a position obtained is to that which is actually expected and thus be able to make the necessary adjustments.

The RD5 robot is used in this work. Currently, its controller is damaged and its recovery is not viable because it uses a Disk Operating System (DOS) operating system. Figure 1 shows the RD5 robot.

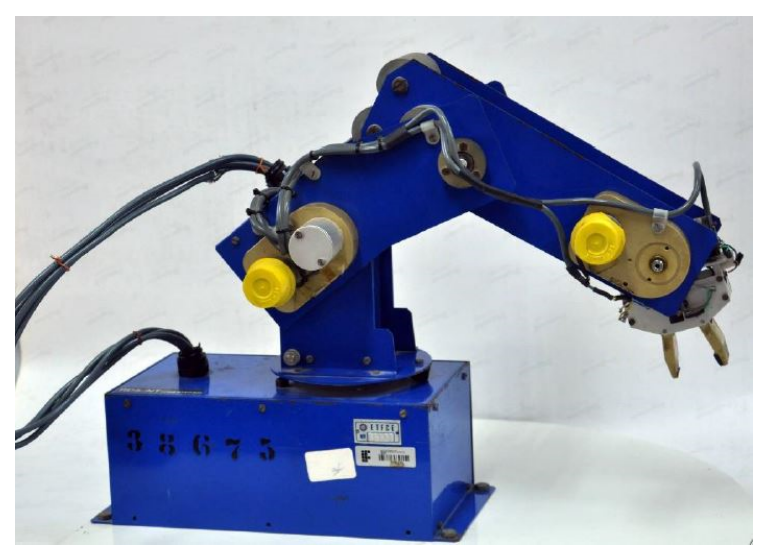

Figure 1: RD5 robot (HONORIO 2016.

The main objective of this work is the modeling of the forward kinematics of the RD5 robot using the Denavit-Hatenberg algorithm and also the construction of its driving and command system. The specific objectives are: fix the damaged connections of the robotic manipulator and replace two potentiometers; develop two auxiliary connection modules, which ensure higher quality and safety to the project's hardware connection scheme; validate the project from a set of collected positioning data, through the analysis of errors between the expected positions and the positions obtained by the robotic manipulator.

This work is divided into five sections. Section 2 describes some related works. Section 3 details the methodology used. Section 4 addresses the results and discussions. For last, Section 5 concludes and summarizes the results of this work.

\section{Related works}

This section analyzes some related works in order to explore the most recent research focusing on the forward kinematics, driving and command system of robotic manipulators.

Iliukhin et al. (2017) developed the Forwards Kinematics (FK) model of a 5 Degrees Of Freedom (DOF) robotic manipulator. The solution of the FK was obtained through DH methods, and the inverse kinematics model was modeled. This work is part of research aimed at creating a robotic manipulator controlled by a Brain-Computer interface.

Liu et al. (2017) designed a 3-DOF robotic manipulator and its kinematic equations using the $\mathrm{DH}$ method. The inverse solution of the kinematics equations was derived, with four joint variables. However, the results were obtained only in a virtual environment. A physical model with all the mechanical difficulties was not made.

Lee, Chiang \& Truong (2018) developed the FK based on DH method for a humanoid walking robot with 22-DOF. The walking includes two states: the right or left foot moving. Each of them has its own initial parameters. When the right foot is moving, the transformation matrix will proceed from the world coordinate frame to the left foot and vice-versa.

Mahmoodabadi \& Ziaei (2019) proposed a DH approach and Jacobi method for each of the arms of a robot in order to gain the kinematic equations of the manipulator. Furthermore, the Lagrange method was utilized to obtain the dynamic equations of motion. Hence, in order to control the dynamics of the robot manipulator, inverse dynamics, and a fuzzy PD controller optimized via particle swarm optimization were used.

Ge et al. (2019) developed a kinematic modeling method based on dual quaternions. The method requires less calculation time comparing to the traditional approaches, e.g. the approach using DH. With this method, the kinematic problem of multi-DOF manipulator is decomposed into several sub-problems.

Gao et al. (2018) developed a parameter identification method based on $\mathrm{DH}$ to decrease the movement uncertainty of industrial robots. Redundant parameters are addressed in the identifier procedure. The kinematic model of a 6-DOF industrial robot was developed and linearized to obtain the parameter identification coefficient matrix. The matrix is not with full rank, which means some parameters in this matrix are linearly dependent. This fact makes the direct identification of unknown parameters unfeasible. To solve this, singu- 
lar value decomposition was used to determine the redundant parameters, which are then removed from the matrix. An alternative identification algorithm with a modified least-square scheme is used to estimate the structural parameters of the robot.

Jamwal et al. (2010) worked on FK mapping of a parallel robot designed for ankle joint rehabilitation treatments. Parallel robots produce non-linear motions hence a unique closed form solution of their FK cannot be obtained. To solve this problem, a modified fuzzy inference system was proposed. Their parameters were optimized using modified genetic algorithm and reached satisfactory accuracy and computation time.

Bearing in mind that none of the articles mentioned above makes use of Euclidian Distance evaluation of errors, this work proposes the use of it to evaluate and compare results.

\section{Methodology}

The first step of project execution is the FK modeling of the RD5 robot with the use of the DH technique. The second part is the development of the driving and command system. The last part is the model testing. The manipulator obeys the programming commands and positions itself in the 3D space. Parallel to this, the FK algorithm shows the expected positioning of the manipulator in each situation and carry out the acquisition of error estimation data.

\subsection{RD5 robot}

The RD5 robot is a mechanical structure formed by an open kinematic chain of links interconnected by joints, controlled by software that uses sensors to be guided through its workspace. Its configuration can be compared with the human anatomy, which includes base, shoulder, elbow, wrist, and end-effector. Different types of end-effector can be added to a robot, depending on the task to be performed. In the case of the RD5 robot, the end-effector is a claw.

\subsubsection{Degrees of freedom}

The number of independent movements that an object can perform in a space is called Degrees Of Freedom (DOF). To completely specify the object in space, in addition to location, orientation is required. Six DOF is desired to position and orient the object, three for spatial positioning and three for orientation (NIKU; BENJAMIN, 2013). There are manipulators with five or less
DOF, such as the RD5 robot that has 5-DOF. For this work, the end-effector is not used, so only 4-DOF is going to be necessary.

\subsubsection{Workspace}

According to Rosário \& Maurício (2005), a robot's workspace is the name given to the various combinations of rotational and linear configurations that can be made from the first three joints of a robot, starting at the base. Depending on the type of joint, rotation $(\mathrm{R})$, or translation or prismatic $(\mathrm{P})$, there are different classes of manipulators. The RD5 robot is a Vertical Articulated type (RRR) because it has its first three joints of the rotational type. This type of joint is able to operate in a larger working space, but it has low mechanical rigidity, complex control, and variable moment of inertia.

\subsubsection{Sensors}

There are several types of sensors used in robotics: position, speed, acceleration, force, pressure, torque, visible and infrared light, touch, and proximity sensors. The RD5 robot uses only position sensors, in this case, a potentiometric sensor.

\subsubsection{Control system}

The mechanical structure of the RD5 robot is poor in precision and moment of inertia. Besides, the positioning error accumulates from joint to joint. These difficulties are overcome thanks to advanced control techniques. A control system consists of controlling the output of a system by comparing it with the reference input; the difference between output and input generates an error signal. The controller handles these signals, amplifying the system's actuators (OGATA; SEVERO. 1998). For the RD5 robot, a Proportional Integrator (PI) controller is used. The gains of this controller were determined based on the work developed by Honorio (2016), who designed a controller for the RD5 robot base joint.

\subsection{Forward Kinematics}

RD5 robot has four rotational joints. The claw (endeffector) is not part of the scope of this work. A servomechanism was developed for each joint. The feedback of the angular position through the potentiometric sensor is responsible for the closed loop system. The DH 
parameters are obtained from the starting position definition. In Figure 2 is shown the schematic drawing of the RD5 robot.

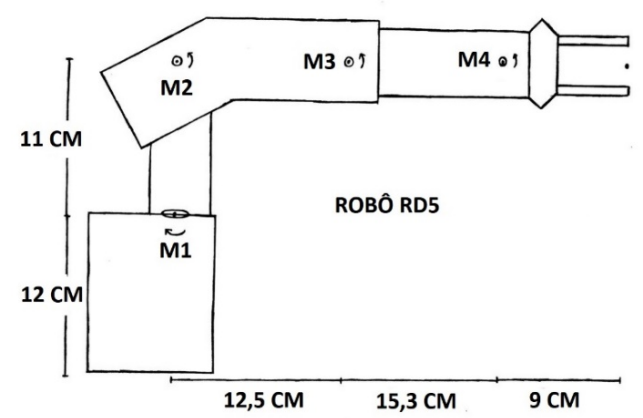

Figure 2: Schematic drawing of the RD5 robot.

The rotation can be done around three different axes. After the development of the internal products that describe the orientation of the reference system in relation to the current system, it is possible to obtain the generic rotation matrices $X, Y$ and $Z$ in relation to an angle $\theta$. The translation has one more coordinate of vectors, as it presents another DOF since the rotation must be defined around an axis, with three possible rotations, therefore it is more complex (SANTOS, 2004).

The link of a manipulator is a rigid element, while a joint connects two links, except for the end of the manipulator that has only one bounding joint. The relationship between links and joints gives rise to very important kinematic parameters in robotics (SANTOS, 2004). A specific symbology helps to understand the definition of these parameters:

- $O_{i}$ : starting point of system with i coordinate.

- $z_{i} \bigcap x_{i}$ : intersection point between $z_{i}$ and $x_{i}$ axes.

- $\overline{O_{l}, P_{l}} \mid x_{i}$ : distance to point $O_{i}$ from point $P_{i}$ measured a long $x_{i}$ axis.

- $\angle\left(x_{i}, z_{i}\right) \mid y_{i}: x_{i}$ steering angle to $z_{i}$ around $y_{i}$.

Thereby, it is possible to obtain the kinematic parameters in robotics, that are:

- Link length: $l_{i}=\overline{\left(z_{l-1} \bigcap x_{l}\right), O_{l} \mid x_{l}}$

- Joint displacement: $d_{i}=\overline{O_{l-1},\left(z_{l-1} \bigcap x_{l}\right) \mid z_{l-1}}$

- Joint angle: $\theta_{i}=\angle\left(x_{i-1}, x_{i}\right) \mid z_{i-1}$

- Link torsion angle: $\alpha_{i}=\angle\left(z_{i-1}, z_{i}\right) \mid x_{i}$

\subsubsection{Denavit-Hartenberg algorithm}

The problem with FK is in finding a relationship between the individual joints and the position and orientation of the end-effector. The $\mathrm{DH}$ algorithm helps to solve these problems (SPONG; HUTCHINSON; VIDYASAGAR, 2004). In Figure 3 is shown the link and joint diagram for the RD5 robot. Through the diagram of links and joints and the use of the DH algorithm it is possible to calculate the parameters of links and joints, which can be seen in Table 1 .

Table 1: DH parameters of links and joints.

\begin{tabular}{lcccc}
\hline$T$ & $\theta$ & $d$ & $l$ & $\alpha$ \\
\hline$T_{0}^{R}$ & $0^{\circ}$ & 12 & 0 & $0^{\circ}$ \\
\hline$T_{1}^{0}$ & $\mathrm{M}_{1}$ & 11 & 0 & $-90^{\circ}$ \\
\hline$T_{2}^{1}$ & $\mathrm{M}_{2}$ & 0 & 12.5 & $0^{\circ}$ \\
\hline$T_{3}^{2}$ & $\mathrm{M}_{3}$ & 0 & 15.3 & $0^{\circ}$ \\
\hline$T_{4}^{3}$ & $\mathrm{M}_{4}$ & 0 & 9 & $-90^{\circ}$ \\
\hline$T_{H}^{4}$ & $0^{\circ}$ & 0 & 0 & $0^{\circ}$ \\
\hline
\end{tabular}

The homogeneous transformation matrix of each joint is then obtained from Equation 1, given by Equations 2 to 7. The multiplication of the homogeneous matrices of each joint gives rise to the final orientation matrix $T_{H}^{R}$, which is represented by Equation 8. To simplify the Equations, cosine is $C$, sine is $S$, and the joint angles are $M 1, M 2, M 3$ and $M 4$.

$T_{i}^{R}=\left[\begin{array}{cccc}C \theta_{i} & -S \theta_{i} C \alpha_{i} & S \theta_{i} S \alpha_{i} & l_{i} C \theta_{i} \\ S \theta_{i} & C \theta_{i} C \alpha_{i} & -C \theta_{i} S \alpha_{i} & l_{i} S \theta_{i} \\ 0 & S \alpha_{i} & C \alpha_{i} & d_{i} \\ 0 & 0 & 0 & 1\end{array}\right]$

$$
T_{0}^{R}=\left[\begin{array}{cccc}
1 & 0 & 0 & 0 \\
0 & 1 & 0 & 0 \\
0 & 0 & 1 & 12 \\
0 & 0 & 0 & 1
\end{array}\right]
$$

$T_{1}^{0}=\left[\begin{array}{cccc}C M_{1} & 0 & -S M_{1} & 0 \\ S M_{1} & 0 & C M_{1} & 0 \\ 0 & -1 & 0 & 11 \\ 0 & 0 & 0 & 1\end{array}\right]$

$$
T_{2}^{1}=\left[\begin{array}{cccc}
C M_{2} & -S M_{2} & 0 & 12.5 C M_{2} \\
S M_{2} & C M_{2} & 0 & 12.5 S M_{2} \\
0 & 0 & 1 & 0 \\
0 & 0 & 0 & 1
\end{array}\right]
$$




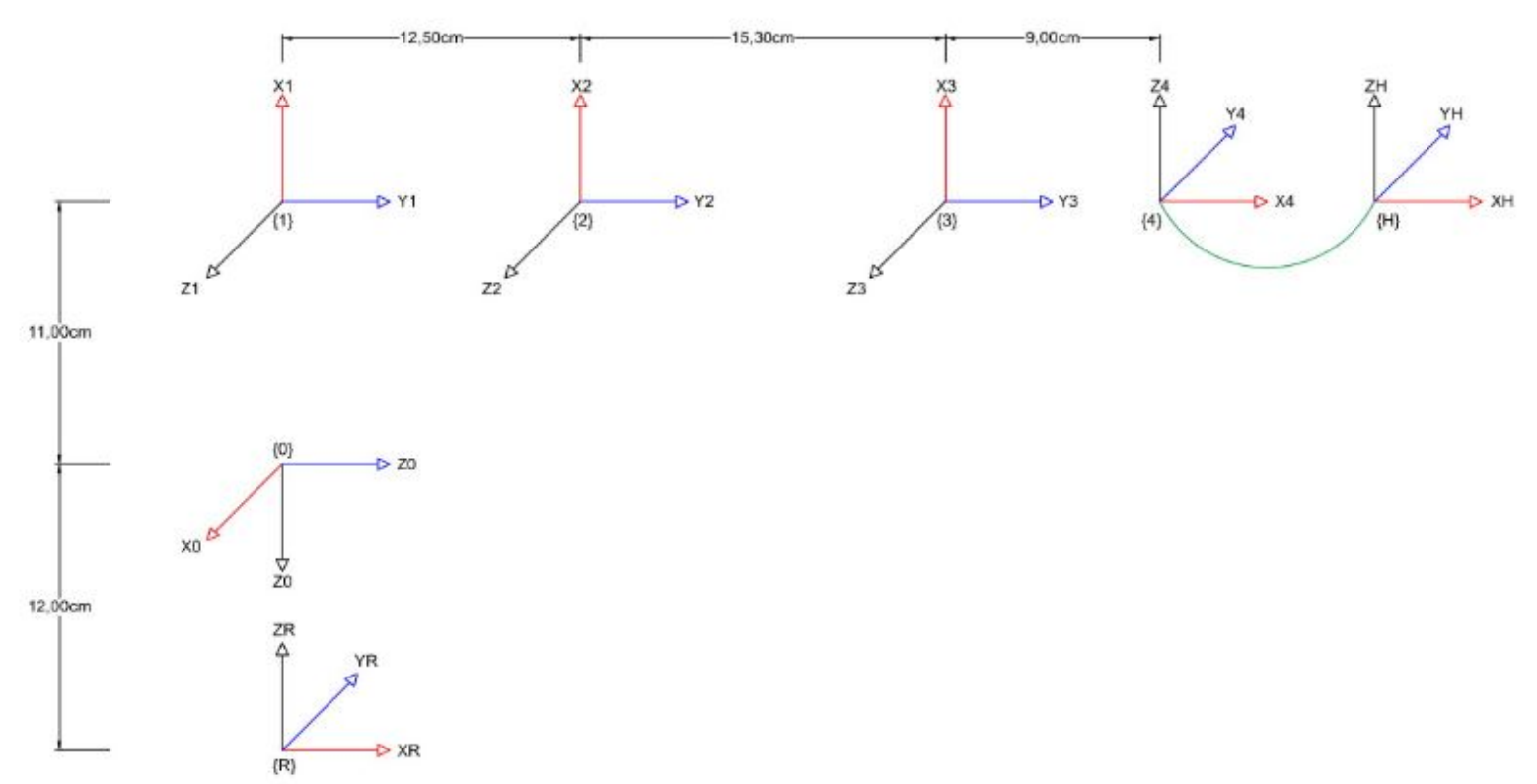

Figure 3: Link and joint diagram for the RD5 robot.

$$
\begin{gathered}
T_{3}^{2}=\left[\begin{array}{cccc}
C M_{3} & -S M_{3} & 0 & 15.3 C M_{3} \\
S M_{3} & C M_{3} & 0 & 15.3 S M_{3} \\
0 & 0 & 1 & 0 \\
0 & 0 & 0 & 1
\end{array}\right] \\
T_{4}^{3}=\left[\begin{array}{cccc}
C M_{4} & 0 & -S M_{4} & 9 C M_{4} \\
S M_{4} & 0 & C M_{4} & 9 S M_{4} \\
0 & -1 & 0 & 0 \\
0 & 0 & 0 & 1
\end{array}\right] \\
T_{H}^{4}=\left[\begin{array}{cccc}
1 & 0 & 0 & 0 \\
0 & 1 & 0 & 0 \\
0 & 0 & 1 & 0 \\
0 & 0 & 0 & 1
\end{array}\right] \\
T_{H}^{R}=T_{0}^{R} T_{1}^{0} T_{2}^{1} T_{3}^{2} T_{4}^{3} T_{H}^{4}
\end{gathered}
$$

\subsection{Interface modules}

Interface modules are used to assemble the driving and command system for the manipulator. These modules serve to bring more precision and stability to the connections between devices. The modules are: facilitator module 1 , responsible for the intermediary between the microcontroller connections and other devices; and the facilitator module 2 , responsible for the connection between the activation and command system and the RD5 robot. In Figure 4 is shown the block diagram of the connections between devices of the drive system and the robotic manipulator.

\subsection{Drive and command system}

The RD5 robot's drive and command system includes an Arduino Mega microcontroller, two L298N power drives, two auxiliary connection modules, four $12 \mathrm{~V} \mathrm{DC}$ motor with speed reduction gearbox, an external voltage source, and an electronic distribution board.

Thus, a complete schematic diagram, shown in Figure 5 was prepared with the purpose of facilitating the understanding of the connections of the drive and command system with the robotic manipulator.

\subsection{RD5 robot positioning equations}

Positioning equations are necessary to control the angular position of motors. The straight-line equation method is used to obtain the angular positioning equation for each axis. The acquisition of data for the points of the line is performed from the measurement of angles in each axis.

The angular position of a given motor is related to the value provided by the potentiometer that is coupled to its axis, in which its reading is made by an analog 


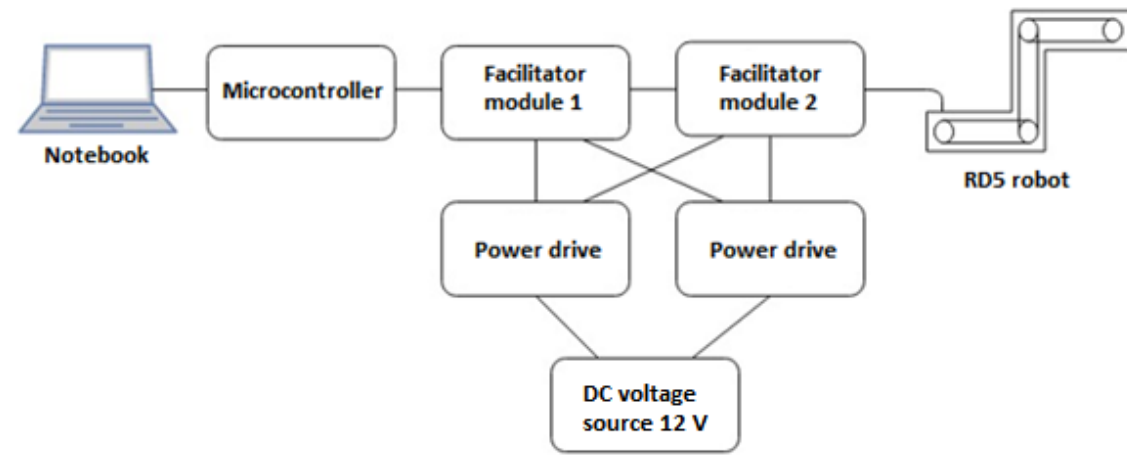

Figure 4: Block diagram of the connections.
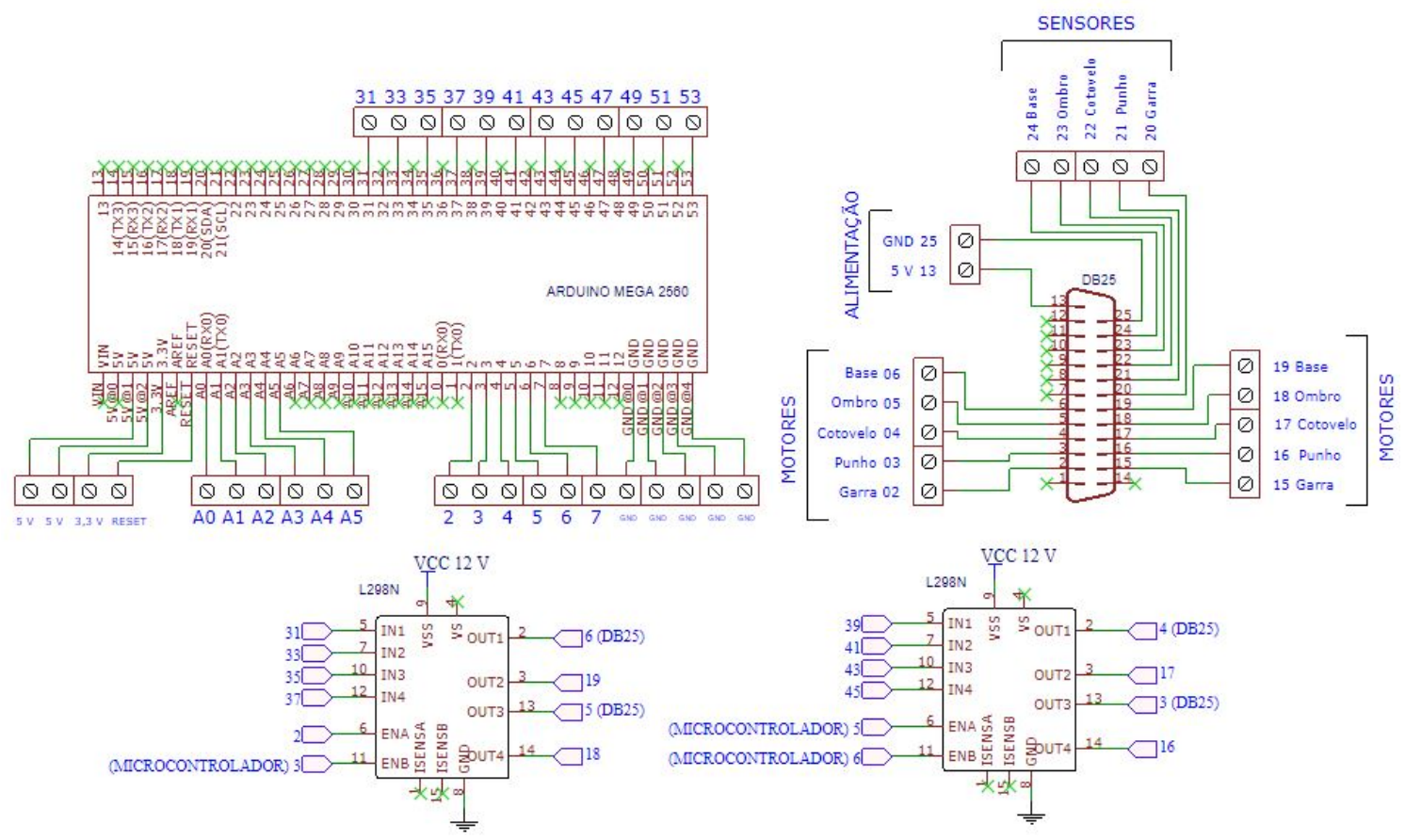

Figure 5: Schematic diagram of driving and command system of the RD5 robot.

pin. When rotating the motor axis to which the potentiometer is attached, a variation in the sensor reading is observed that varies within the range of 0 to 1023 bits. This variation occurs because the resolution of the microcontroller is 10 bits. Thus, there is a proportion of 0 $\Omega$, which equals 0 bits, and $10 \mathrm{~K} \Omega$, which equals 1023 bits.

Values of acceptable error in bits were settled for each robot joint. This margin of error is imposed to avoid a problem of positioning instability in the robot, such as vibration. These errors vary from joint to joint due to problems with low mechanical stiffness, lack of lubrication, and different potentiometers. In Table 2 is shown the acceptable error values for each joint of the robotic manipulator.

Even with the use of a $P I$ controller based on the work of Honorio (2016), there is still some instability problem. The gains of the controller were determined from the gains for the RD5 robot base joint, and then these gains were applied to the other joints of the manipulator. Consequently, the most stable joint is the base. The gains are: $P=12$ and $I=0.0156$. 
In straight line equation, the $x$ axis comprises the two angles chosen at each joint for the positioning calculation. The $y$ axis comprises the value read in bits by the potentiometer for each joint angle. The Equations for all joints position can be seen in Table 3

Table 3: Position equations.

\begin{tabular}{lc}
\hline Joint & \multicolumn{1}{c}{ Equation } \\
\hline Base & $\mathrm{y}=-3.04 \mathrm{x}+821.6$ \\
\hline Shoulder & $\mathrm{y}=3.33 \mathrm{x}+136.3$ \\
\hline Elbow & $\mathrm{y}=5.33 \mathrm{x}+80.3$ \\
\hline Wrist & $\mathrm{y}=-3.55 \mathrm{x}+859.5$ \\
\hline
\end{tabular}

\section{Results and discussions}

This chapter presents the results and discussions. Through the result of the orientation matrix $T_{H}^{R}$, the expected orientation of the end-effector in axes $x, y$ and $z$ is known from the angles imposed for each motor. The end-effector position measurement is compared with the results of the DH algorithm. Thirty-nine positions were chosen for the analysis of results.

To calculate the position error, which is the difference between the expected position and the one obtained, the Euclidean Distance technique is used, which can be understood through Equation 9

$$
E r r o r=\sqrt{(X \exp -X o b t)^{2}+(Y \exp -Y o b t)^{2}+(Z \exp -Z o b t)^{2}}
$$

Table 2: Acceptable error for each joint

\begin{tabular}{lc}
\hline Joint & Acceptable error in bits \\
\hline Base & 2 \\
\hline Shoulder & 5 \\
\hline Elbow & 10 \\
\hline Wrist & 10 \\
\hline
\end{tabular}

In Figure 6 is shown the error levels in each expected position. Light blue represents errors between 0 and $0.69 \mathrm{~cm}$; blue represents errors between 0.7 and $1.1 \mathrm{~cm}$, orange represents errors between 1.12 and 1.35 $\mathrm{cm}$, and red represents errors greater than $1.36 \mathrm{~cm}$. The $3 \mathrm{D}$ graph was divided into three other $2 \mathrm{D}$ graphs that facilitate viewing in certain positions.

There are errors in all positions that can be explained by some factors. The RD5 robot has mechanical problems that influence errors. There are gaps in all joints. Even with the tightening of the screws, the problem has not been completely corrected. It was not possible to disassemble the robot for better lubrication and cleaning. Two sensors were placed with lower quality and precision than the factory ones. An adaptation was made to fix one of the potentiometers in order to be able to carry out the tests. The gains of the PI con-

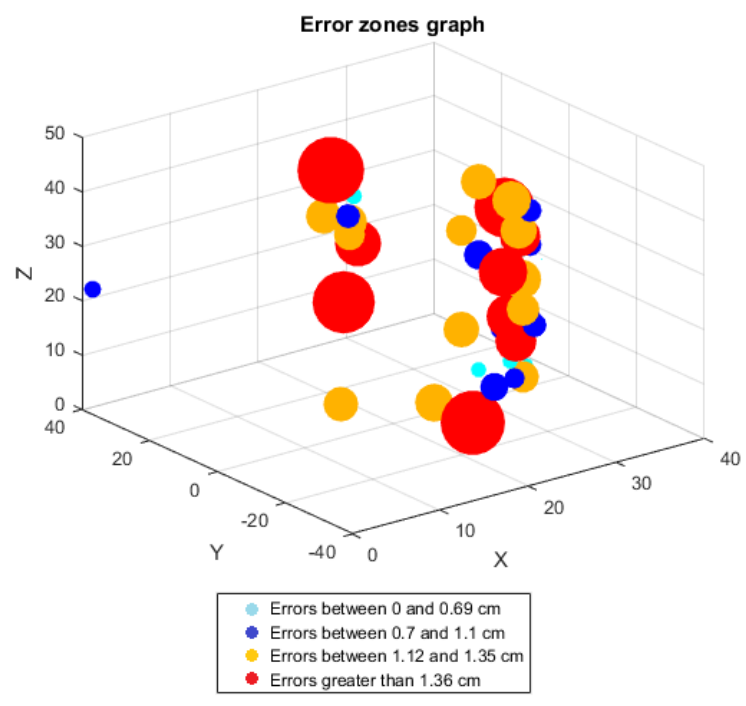
troller only minimized the instability and vibration for the RD5 robot joints.

The graph in Figure 7 a) shows the points on $x$ and $z$ axes, respectively, distance and height in relation to the origin. It is concluded that the shorter the distance from the origin, the greater the position error. The jus- 
Development of forward kinematics and construction of the driving and command system of a robotic manipulator

tification is that in these positions, the joints bend, and the error accumulates.

In Figure 7(b) are $y$ and $z$ axes, which are the left or right side and height, respectively. An increase in error is observed when the end-effect points to extreme directions and with greater height. A justification for increasing the position error is that the errors accumulate due to the acceptable error for each joint.

In Figure 7.c) are $x$ and $y$ axes, which are respectively distance and left or right in relation to the origin. The further to the sides, the greater the tendency to increase position error. Once again, the justification is given by the accumulation of the position error due to the acceptable error in bits for each joint.

At the end of the analysis of all the error zone graphs, the conclusion is that a set of factors leads to an increase in the position error:

- height increase in relation to the plane's origin;

- shortening the distance from the plane's origin;

- further to the left or right side of the RD5 robot;

- accumulation of the acceptable error in joint bits.

However, there are some exceptions that do not fit this set of factors.

The mean error per position is $0.56 \mathrm{~cm}$. The $x$ and $z$ axes mean error are slightly higher than the mean error per position, while the $y$ axis mean error is less than the mean error per position. These differences can be explained by the fact that $x$ (distance) and $z$ (height) axes suffer more from the robot's instability due to the looseness and the weight of its links, while in $y$ axis (left or right direction) is more influenced by the base joint, where $P I$ controller generated greater stability. This advantage of the $y$ axis in relation to the $x$ and $z$ axes is shown in the expected and obtained positions graphs from Figures 8,9 , and 10

Despite the errors, which are already expected due to the mechanical problems of the RD5 robot, the project proved to be satisfactory for the forward kinematics model operating in conjunction with the robotic manipulator drive and command system.

\subsection{Future prospects}

Further researches is necessary to find out new approaches or improve the existing ones in the area of robotic manipulators. Future prospects can allow the total use of robotic systems in varied essential practices for society; they are:
- Application of closed-loop digital control systems to all robot joints;

- Development of a trajectory system through inverse kinematics;

- Use of remote control techniques, for example, mobile application;

- Implementation of instrumentation techniques, such as proximity sensors to an obstacle.

\section{Conclusions}

The project started with the development of the forward kinematics through the Denavit-Hartenberg method. So, the driving and command system was built. The results were obtained when the robot obeyed the commands and positioned itself in the 3D plane.

The study of the positioning error was done using Euclidian Distance through the position measurement obtained, and compared with the expected position data acquired by $\mathrm{DH}$ algorithm. The position mean error of $0.56 \mathrm{~cm}$ is acceptable, considering the number of factors limiting the project: height increase in relation to the plane's origin; shortening the distance from the plane's origin; further to the left or right side of the RD5 robot; and accumulation of the acceptable error in joint bits.

The concepts for the execution of FK and driving and command system for the robotic manipulator used in this work address the three main branches of mechatronics engineering, which are mechanics, electronics, and computing. Mechanics fit into the structural and measurement part of the robot, electronics appear in the entire process of instrumentation, application, and development of circuit boards and computation complements the work with command programming and analysis of the results.

All this amount of information, coming from different sources of study, makes an integration of great value, which is positive for the choice of the topic in the area of robotics. The expected objectives were achieved, and the results were satisfactory.

\section{Acknowledgements}

The author thanks IFCE (Federal Institute of Ceará) and LIR (Robotics laboratory) for the academic support and FUNCAP (Fundação Cearense de Apoio ao Desenvolvimento Científico e Tecnológico) for the financial support. 


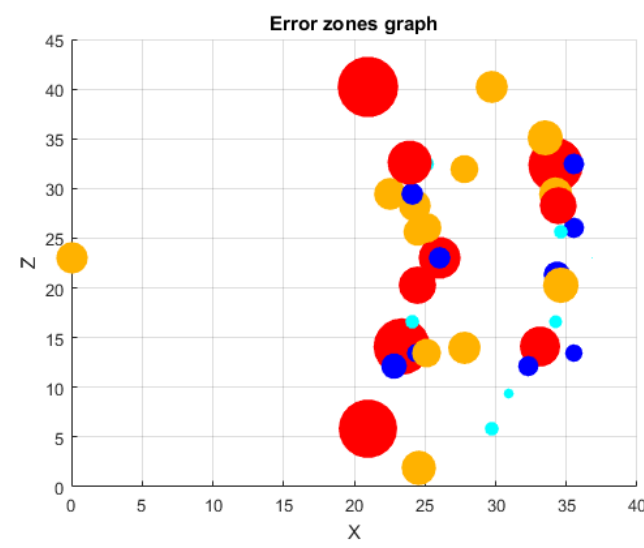

(a)

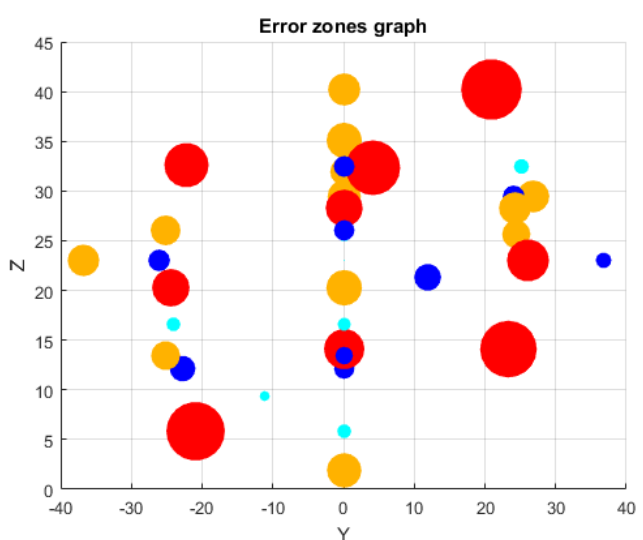

(b)

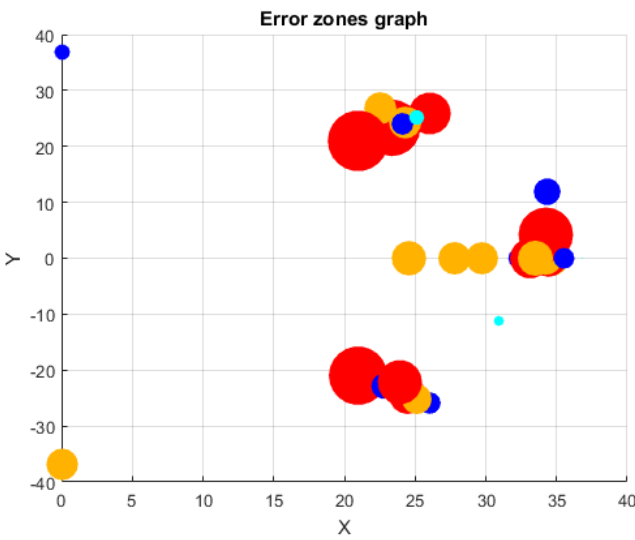

(c)

Figure 7: Error zones.

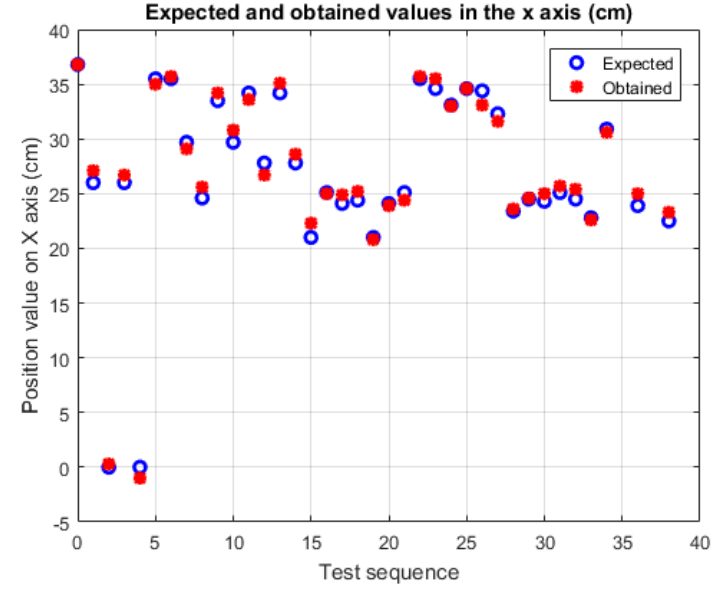

Figure 8: Expected and obtained positions in the $\mathrm{x}$ axis.

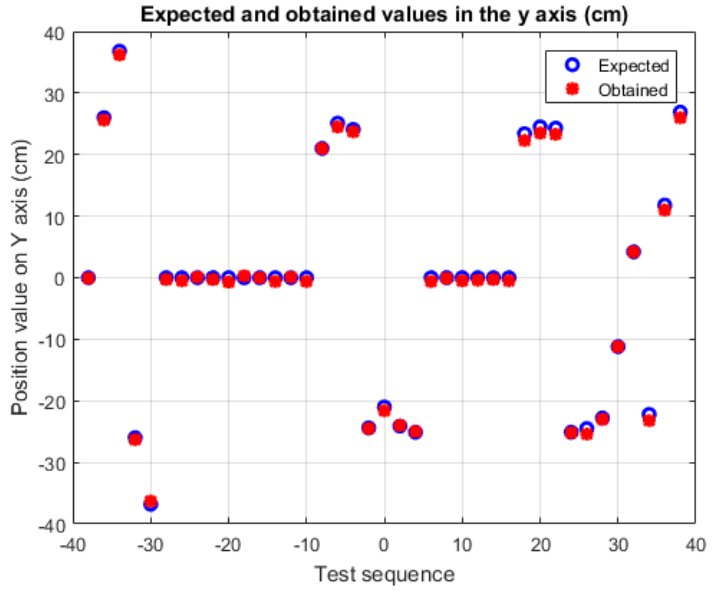

Figure 9: Expected and obtained positions in the y axis. 
Development of forward kinematics and construction of the driving and command system of a robotic manipulator

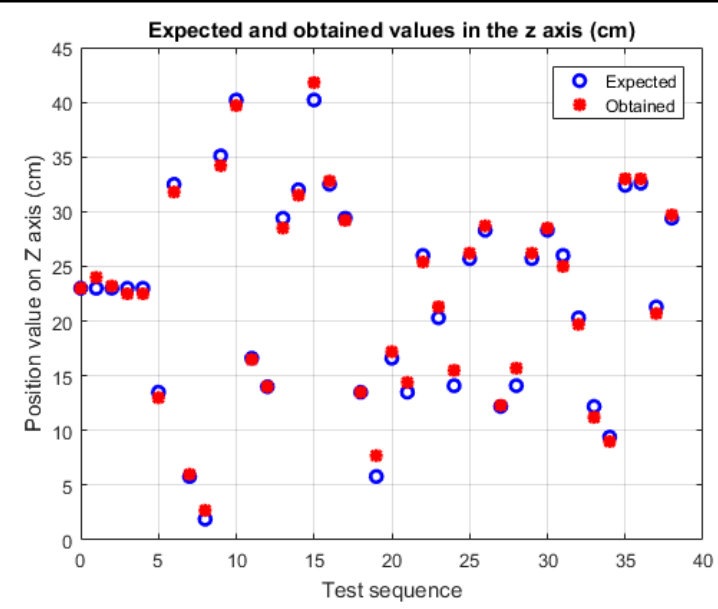

Figure 10: Expected and obtained positions in the $\mathrm{z}$ axis.

\section{Conflicts of Interest}

There is no conflict of interest.

\section{References}

GAO, G.; SUN, G.; NA, J.; GUO, Y.; WU, X. Structural parameter identification for 6 dof industrial robots. Mechanical Systems and Signal Processing, Elsevier, v. 113, p. 145-155, 2018.

GE, W.; CHEN, L.; WANG, X.; XING, E.;

ZIELINSKA, T. Kinematics modeling and analysis of manipulator using the dual quaternion. In: IEEE. 2019 IEEE International Conference on Mechatronics and Automation (ICMA). [S.1.], 2019. p. 750-755.

HONORIO, A. A. Projeto e implementação de reforma e atualização (Retrofit) de sistema de comando e controle de braço robótico manipulador didático. Dissertação (Undergraduation in Mechatronics Engineering) — Instituto Federal do Ceará - IFCE, Fortaleza, Brazil, 2016.

ILIUKHIN, V.; MITKOVSKII, K.; BIZYANOVA, D.; AKOPYAN, A. The modeling of inverse kinematics for 5 dof manipulator. Procedia Engineering, Elsevier, v. 176, p. 498-505, 2017.

JAMWAL, P.; XIE, S.; TSOI, Y.; AW, K. Forward kinematics modelling of a parallel ankle rehabilitation robot using modified fuzzy inference. Mechanism and Machine Theory, Elsevier, v. 45, n. 11, p. 1537-1554, 2010.
LEE, K.-C.; CHIANG, P.-Y.; TRUONG, H.-A. The forward kinematics for the hr1-nfu humanoid robot. In: IEEE. 2018 IEEE International Conference on Advanced Manufacturing (ICAM). [S.1.], 2018. p. 455-458.

LIU, F.; GAO, G.; SHI, L.; LV, Y. Kinematic analysis and simulation of a 3-dof robotic manipulator. In: IEEE. 2017 3rd International Conference on Computational Intelligence $\&$ Communication Technology (CICT). [S.1.], 2017. p. 1-5.

MAHMOODABADI, M. J.; ZIAEI, A. Inverse dynamics based optimal fuzzy controller for a robot manipulator via particle swarm optimization. Journal of Robotics, Hindawi, v. 2019, 2019.

MITTAL, R.; NAGRATH, I. Robotics and control. [S.1.]: Tata McGraw-Hill, 2003.

NIKU; BENJAMIN, S. Introdução à robótica: Análise, Controle e Aplicações. [S.1.]: Rio de Janeiro: LTC, 2013.

OGATA, K.; SEVERO, B. Engenharia de controle moderno. [S.1.]: Prentice Hall do Brasil, 1998.

ROSÁRIO; MAURÍCIO, J. Princípios de mecatrônica. Pearson Prentice Hall, 2005.

SANTOS, V. M. F. Robótica Industrial.

Departamento de Engenharia Mecânica, Universidade de Aveiro, 2004. 2004. Disponível em: <http://www.ece.ufrgs.br/ rventura/ RoboticaIndustrial.pdf> Acesso em: 24 jan. 2018.

SPONG, M. W.; HUTCHINSON, S.; VIDYASAGAR, M. Forward kinematics: the denavit-hartenberg convention. Robot Dynamics and Control, 2nd ed.; John Wiley \& Sons: New York, NY, USA, p. 57-82, 2004. 